\title{
LOS TRANSTORNOS DEL MOVIMIENTO, DE LA POSTURA Y DEI. TONO POR PATOLOGIA EXTRAPIRAMIDAL
}

\author{
L. BARRAQUER-Bordas *
}

Los sistemas extrapiramidales no constituyen una agrupación positivamente definida. Representan solamente un conjunto de estructuras de función esencialmente eferente, con sus interconexiones $y$ sistemas de proyección, aparte del sistema piramidal, esto es, del sistema que incluye la proyección orto-piramidal y el componente yuxtapiramidal inhibidor del reflejo miotático.

Así delimitados, los sistemas extrapiramidales, aunque se centran sobre unos núcleos grises de posición profunda, tienen también un eslabón cortical constituído por sistemas neuronales de la corteza cerebral que envian proyecciones a aquellos núcleos profundos. Tales núcleos son principalmente el núcleo caudado, el núcleo lenticular, el núcleo subtalámico o cuerpo de Luys y la substancia nigra o locus niger. En el núcleo lenticular se distingue una parte interna llamada pallidum y una parte externa denominada putamen. Funcionalmente, el putamen se aproxima al núcleo caudado, formando una totalidad denominada striatum.

El conjunto constituído por estas masas grises mantiene conexiones principalmente con el cerebelo, la formación reticulada, el tálamo óptico, la corteza cerebral e el hipotálamo. Las conexiones con el cerebelo son principalmente aferentes. a través del centro mediano del tálamo y más directamente (Carpenter). Las conexiones con la formación reticulada son complejas: por una parte, los sistemas reticulares inespecificos dejan sentir su acción sobre el striatum a través del centro mediano del tálamo; por otra parte, el pallidum proyecta su influencia sobre la formación reticulada, sino directamente, si al menos a través de un circulio pálido-tálamo-córtico-reticular. Las conexiones talámicas son asimismo complejas. Esquemáticamente van del tálamo al striatum y vuelven del pallidum hacia el tálamo: las primeras arrancan del centro mediano, mientras que las últimas van a parar a los núcleos talámicos que Hassler y col. denominan ventro-oral anterior y látero-polar. También habría proyecciones del tálamo sobre el pallidum (DennyBrown). Las relaciones con la corteza cerebral tienen aún mayor complejidad.

Conferencia pronunciada durante el cursillo intensivo de Actualidades de Neurologia, organizado por el Dr. R. Sales-Vásquez en la Clínica Médica Universitaria del Prof. A. Pedro-Pons, de Barcelona, en noviembre de 1964.

* Departamento de Neurologia de la Clínica Universitaria de Patología General y Escuela de Neurologia del Hospital de la Sta. Cruz y San Pablo, Barcelona. 
La corteza precentral envia proyecciones al striatum y hay también proyecciones corticales sobre la formación reticular. Por otra parte, la corteza recibe una proyección indirecta de impulsos estrio-palidales, a través del tálamo. Las conexionés de los núcleos basales con el hipotálamo nos interesan menos.

Por sua parte, las cuatro grandes masas grises extrapiramidales tienen sus propias conexiones entre ellas. El striatum se proyecta sobre el pallidum y sobre la substancia nigra. El pallidum envía sus fibras al tálamo $\mathrm{y}$ al núcleo subtalámico de Luys, el cual, a su vez, se proyecta sobre el propio pallidum. También la substancia nigra enviaría fibras al pallidum, pero las conexiones eferentes de esta masa nuclear son poco conocidas.

La consideración sintética de estas conexiones permite algunos comen tarios de conjunto: a) no puede definirse el sistema extrapiramidal como un conjunto exclusivamente subcortical, pues la doble conexión, aferente y eferente, con la corteza es importante; b) el pallidum aparece como el "centro" más importante dentro del dispositivo extrapiramidal, atuando como punto nodal en la recepción y en el envío de impulsos; c) el tálamo óptico mantiene conexión muy estrecha con el pallidum; d) el striatum se situa en un circuito complejo y múltiple que parece ser ante todo córtico-stríopálido-tálamo-cortical. Pero también recibe impulsos del cerebelo y de la formación reticulada $\mathrm{y}$, por otra parte, desde su segundo eslabón cortical, los impulsos pueden derivar hacia el sistema piramidal o hacia sistemas no piramidales (proyecciones córtico-reticulares) y, de este modo, pueden llegar a los niveles segmentarios tanto por la vía piramidal como por los sistemas retículo-espinales. Añadiremos que, por lo que se va conociendo y especulando (Bucy, Jung y Hassler), el striatum parece jugar, probablemente a través de este circuito, una importante función de inhibición.

Además de los núcleos capitales mencionados, hacia la parte alta del mesencéfalo se sitúan unos sistemas de gran importancia en la organización de la motilidad, pues rigen integraciones en relación con los movimientos de elevación y descenso de la cabeza y de giro y de desviación hacia uno y otro lado (Hess y Hassler). Los movimientos de los ojos siguen siempre o casi siempre a los de la cabeza.

\section{LOS FENOMENOS CLINICOS}

La patología de los sistemas extrapiramidales se expresa por dos grandes órdenes de transtornos: movimientos espontáneos anormales y transtornos del tono muscular. Por lo demás, es muy difícil situar un límite entre unos y otros, pues, p. ej. las distonías de actitud son un transtorno del tono muscular que comporta una discinesia espontánea. En otras ocasiones, la discinesia aparece ligada a un cierto acto, p. ej. en los calambres profesionales. Finalmente, no puede descuidarse de la hipocinesia primaria de algunos pacientes extrapiramidales. Y aun deberemos referirnos al capítulo bien singular de las cinesias paradójicas.

A) Movimientos espontáneos anormales o hipercinesias - El concepto de movimiento espontáneo anormal, aparentemente banal, resulta en realidad difícil de precisar. En primer lugar en lo tocante al término de mo- 
vimientos con que se les distingue hay que tener presente que muchas veces comportan un acompañamiento tónico o, incluso, son en sí mismos de un ritmo tan lento; que suscitan necesáriamente la problemática diferenciación entre hipercinesia $\mathrm{y}$ distonia. Por lo que hace referencia al calificativo de espontáneos, implica en este caso la acepción obvia de "no deseados", esto es de que no son propiamente voluntarios, si bien muchas veces la hipercinesia se precipita en ocasión de un movimiento voluntario o, cuando menos, aumenta durante él. Por lo demás, si se llevase a un extremo tal calificativo de "espontáneos", resultaria no ser más que una abstracción, ya que tales hipercinesias están necesáriamente integradas en el seno del "espiral figural" sensación-movimiento y, por lo tanto, están sujetos a la acción desencadenante o moduladora de toda clase de aferencias. Cabalmente, la concepción fisiopatológica reciente de Denny-Brown subraya esta relación entre estímulos muy variados y la génesis o el curso de las hipercinesias. Y todavía hay que resaltar que siendo "espontáneos" en el sentido que se quiere decir, los movimientos anormales por patología de los sistemas extrapiramidales deben diferenciarse netamente de otros movimientos igualmente anormales y no voluntarios, pero que muestran una dependencia más primaria y grosera en cuanto a sua determinación sensitiva o sensorial. Nos referimos a los reflejos y a los automatismos anormales, por ej., para encontrar un paradigma que interese a estos dos campos, a los reflejos de automatismo medular.

Los movimientos espontáneos anormales por patología extrapiramidal, en suma, son cinesias que nacen más allá de la motilidad voluntaria, a la que pueden desbordar, por así decirlo, y que, aun que guardan una relación de dependencia con las estimulaciones aferentes, ofrecen muchas veces un grado de complejidad y de abigarramiento que traduce una integración neural siempre más complicada que la que rige las respuestas más elementales, que calificamos de reflejos anormales.

Asi delimitado, aunque con cierta imprecisión, el ámbito de tales movimientos, importa decir que los tipos semiológicos que más cumplidamente caben en él son la atetosis y la corea, esta última con el aditamento de los balismos. La situación de los tics es mucho más imprecisa. En cuanto a las mioclonías, parecen responder a la descarga de sistemas reticulares situados a diferentes alturas, puesta en marcha por situaciones morbosas de topografía variada. Su relación con la patología extrapiramidal es, por tanto, mucho más laxa. En lo tocante a los temblores, solamente el de reposo es expresión de la afectación de los sistemas habitualmente calificados de extrapiramidales.

1) Corea - Los movimientos coreicos son irregulares y desordenados, agitan a tal o cual segmento del cuerpo, sin ningún ritmo, sin ninguna propagación determinada. Habitualmente se trata de una serie contínua de movimientos, sin finalidad, de la mano y de la muñeca, de los labios, de la cara, de la lengua, de la parte distal de las extremidades inferiores. Son bruscos, breves y rápidos y de amplitud variable. Carecen de sincronía y aún de simetría, caso de ser bilaterales. Su estudio electromiográfico 
(Herz) ha demostrado que ponen en marcha agrupaciones musculares funcionalmente sinérgicas y que, en la gran mayoría de casos, no entran en juego simultáneamente músculos antagonistas. Habitualmente cursan sobre un fondo de hipotonía y sin despertar un incremento acompañante del tono muscular. Cabalmente, en la corea minor o de Sydenham es muy característica la hipotonia de fondo, con gran aumento de la pasividad. Caracteriza a la corea, la excesiva fluidez cinética con pérdida total de la postura en sentido dinámico (Denny-Brown). Por lo demás, la hipercinesia coreica interfiere y pervierte el curso del movimiento voluntario al conferirle inusitada brusquedad y rapidez (Espadaler). Los movimientos coreicos, como ccurre con la generalidad de hipercinesias extrapiramidales, aumentan con la emoción y ceden durante el sueño.

2) Hemibalismo - Muy próximo a la corea, se caracteriza por movimientos amplios, bruscos, como de lanzamiento de toda una extremidad. Su distribución es hemilateral. Puede afectar a una sola extremidad (monobalismo). Según Diersen y Gioino la diferencia entre corea $\mathrm{y}$ balismo es meramente cuantitativa.

3) Atetosis - Se trata de movimientos ondulantes, que predominan en las extremidades, de ritmo lento, habitualmente repetitivos. La mano es la parte más frecuentemente afectada, luego los territorios faciales, más raramente el pié. En la mano, la hipercinesia se caracteriza por el paso fluctuante, de una hiperextensión de los dedos, frecuentemente asociada a pronación, a una flexión de los mismos, habitualmente acompañada de supinación. En la atetosis pueden ser distinguidas dos o más posturas habituales (Denny-Brown) entre las que fluctua la hipercinesia. Durante el reposo el enfermo atetósico suele permanecer hipotónico, pero la actividad motora determina frecuentemente la aparición de cierto grado de actividad distónica en los músculos de las extremidades superiores, cara y tronco. Para Denny-Brown existiria una transición desde la atetosis hacia el espasmo móvil (o distonia muscular deformante) y de ésta hacia una distonia fijada. Electromiográficamente se ha puesto de manifiesto, en la atetosis, la existencia de una actividad simultanea e irregular de agonistas y antagonistas, variando cuantitativamente en unos y otros, según los momentos (Hoefer, Putman y Herz).

4) Tics - Son actos coordinados, discontínuos, repetidos, bruscos, rápidos y de carácter clónico, estereotipados, que interesan un grupo de músculos que entran en acción a la vez, simultáneamente. Se acepta una base extrapiramidal para la gran enfermedad de los tics (Gilles de la Tourette) y puede discutirse para otras formas, pero la mayoria de tics, principalmente en niños, obedecen a transtornos emocionales, más o menos profundos.

5) Mioclonias - Son contracciones bruscas que interesan a un fascículo muscular, a un músculo o a una serie de músculos. En su acepción más pura, las mioclonías son individuales y autónomas, si bien músculos muy alejados entre si pueden ser afectados simultáneamente (mioclonias 
síncronas). La mioclonía puede ser estimada como una sacudida o contracción rápida e imprevisible de uno o más músculos, distinguible de la ccrea por su carácter abrupto y breve (Denny-Brown). Según Krebs las mioclonias propiamente dichas son anárquicas, mientras que deben calificarse de clonias las contracciones que se agrupan siguiendo las sinergías funcionales normales.

Según este autor, las mioclonias verdaderas, globales y asinérgicas son raras, aunque típicas de un sindrome infrecuente, el paramioclono muiltiple de Friedreich (mioclonías asinérgicas, asíncronas y arrítmicas). Las mioclonias de la encefalitis epidémica son asinérgicas, pero síncronas y ritmadas (Krebs). Se situarían, pues, entre las formas más típicas y las clonías (sinérgicas). Las excitaciones externas cutáneas, musculares y principalmente sensoriales pueden tener un efecto desencadenante, a veces muy neto, sobre las mioclonias.

En algunos cuadros clínicos pueden presentarse asociadas a epilepsia, tanto clonias, como mioclonias auténticas, como ambas clases de fenómenos. Es preciso distinguir cuidadosamente la epilepsia mioclónica degenerativa de Unverritch-Lundborg, de curso progresivo, de las descargas mioclónicas simples de otras formas de epilepsia, tanto si se mantienen dentro de la llamada epilepsia centroencefálica como si se les supone un origen reticular más bajo.

6) Temblores - Fueron caracterizados por Dejerine como oscilaciones involuntarias aparentemente ritmicas, descritas por todo el cuerpo o por una parte de él alrededor de su posición de equilibrio. Bonduelle hace notar que si bien esta definición presupone la regularidad del ritmo, no pueden eliminarse del cuadro de los temblores movimientos menos regulares en los que el ritmo puede resultar roto por bruscas oscilaciones.

Los temblores pueden ser distinguidos en una serie de variedades de acuerdo con sus circunstancias de aparición respecto a la actividad neuromuscular (Bonduelle) :

a) Temblor de reposo - Aparece durante la ausencia de actividad motora. Es el temblor parkinsoniano clásico con un ritmo de 4 a 6 contracciones por segundo, relativamente lento y particularmente regular, desapareciendo durante el movimiento voluntario. De uno a otro segmento, este temblor es asincrónico.

b) Temblores de acción - Se oponen al anterior por su ausencia durante el reposo y su aparición en el curso del acto motor. Pueden distinguirse dos variedades, según su circunstancia de aparición: temblor cinético $o$ de movimiento, movimiento realizado irregularmente más que un verdadero temblor (Bonduelle), es el temblor de la patología cerebelosa que, al final del movimiento, puede continuarse con un temblor estático que cede pronto; el temblor de actitud, denominado también "discenesia volicional de actitud", aparece durante el mantenimiento voluntario de una actitud (Bonduelle), predominando habitualmente en la extremidad distal. Se afectan electivamente las extremidades superiores. Desaparece con el reposo, 
a diferencia del temblor parkinsoniano. El ritmo es más lento que el de este último. La amplitud aumenta mientras va manteniéndose la actituá. El movimiento voluntario se realiza sin irregularidar cuando es algo rápido a diferencia de lo que ocurre en el denominado temblor cinético. Una de las actitudes electivas para la producción de este temblor es la de oposición de ambos índices sin tocarse, manteniendo las manos frente a la cara, con los brazos elevados a la altura de los hombros. El temblor de actitud es muy frecuentemente un temblor "esencial", a veces heredofamiliar, escasamente evolutivo. Esta descripción matizada de las formas de temblor en relación con los actos motores, es más penetrante que la mención simple de un temblor "intencional".

c) Temblor vibratorio - Es muy parecido al temblor fisiológico de la fatiga o emoción. Bonduelle lo describe así: Intermitente o permanente, puede existir en reposo, pero habitualmente no aparece más que en el curso del acto y muy particularmente durante el mantenimiento de una actitud. Predomina en los miembros y en las extremidades distales, molestando más para los pequeños gestos delicados que para los grandes movimientos. Puede ser generalizado (temblor de la parálisis general) afectando entonces a los músculos de la cara y de la lengua. Sus oscilaciones son rápidas, de 8 a 9 por segundo y de amplitud irregular, habitualmente escasa. Puede ponerse de manifiesto en la prueba de los brazos extendidos con los dedos separados, reposando sobre ellos una hoje de papel. Las formas etiológicoclínicas son muy variadas (parálisis general progresiva, hipertiroidismo, alccholismo crónico).

B) Transtornos del tono muscular - Son habitualmente hipertonías o distonias, mas raramente hipotonias.

1) Rigidez parkinsoniana - Como la piramidal, la hipertonía extrapiramidal aparece en virtud de un proceso de liberación de las actividades miotáticas, pero esta liberación difiere semiológicamente por una série de características que atañen tanto a la topografía como a la calidad de la manifestación hipertónica.

Desde el punto de vista topográfico, la hipertonía extrapiramidal es de distribución más global que la piramidal. No predomina en los músculos de mayor uso voluntario ni tampoco, sensiblemente, en los antigravitatorios. En todo caso, es más acentuada en los de empleo preferentemente automático $\mathrm{y}$ en los proximales.

Por su calidad, la hipertonía extrapiramidal no es elástica, sino plástica, esto es, se mantiene igual o casi igual desde el principio al final de la realización de un movimiento pasivo y no hay la tendencia a volver a la posición de partida (rigidez cerea), excepto cuando se hace muy exagerada e invencible.

Los reflejos locales de postura están típica y electivamente exaltados. La movilización pasiva de las articulaciones, con las elongaciones musculares que provoca, es capaz de poner de manifiesto en muchos casos de hipertonia extrapiramidal - de la cual la rigidez parkinsoniana es el ejemplo 
más asequible - el fenómeno de la "rueda dentada" (C. Negro). Se aprecia entonces que el movimiento pasivo no se realiza de una sola vez, sino mediante interrupciones o sacudidas múltiples, intermitentes, más o menos regulares, que representam un rápido aumento de la resistencia a la elongación muscular, seguido de súbita disminución y que se repiten, sensiblemente iguales entre sí, hasta el fin del movimiento. Tal fenómeno produce la impresión de que la movilidad de la articulación está regulada por un mecanismo de ruedas de engranaje.

Desde el punto de vista semiológico la rigidez parkinsoniana puede ser descrita como una disminución electiva, acentuada y plástica de la pasividad (André-Thomas) que expresa fisiopatológicamente, um aumento electivo, acentuado y plástico de la "reacción de oposición" (André-Thomas) o antagonismo neuromuscular local o miotático. "La aplicación de las pruebas de pasividad al estudio de la rigidez parkinsoniana es la más demostrativa" (André-Thomas y De Ajuriaguerra). El balanceo brusco que es posible imprimir a la mano sacudiéndola por la muñeca, p. ej., está disminuído. El penduleo pasivamente imprimido a las piernas del paciente, estando éste sentado y pendiendo libremente aquéllas, está limitado (Wartenberg).

El estudio instrumental de los reflejos musculares clínicos (Tinel y col.) revela que en el parkinsonismo existe una contracción exagerada de los antagonistas, puesta en marcha por la provocación del reflejo de los agonistas. Esta exageración es mayor cuando se trata de los antagonistas de los músculos antigravitatorios, p. ej., del tibial anterior al provocar el reflejo fásico del tríceps sural (habitualmente llamado reflejo aquíleo).

La exaltación de la actividad miotática propia de la rigidez parkinsoniana comporta el establecimiento de un tipo singular de "desintegración de las funciones motoras", caracterizado por dos órdenes de fenómenos (Walshe, Barraquer-Bordas): fenómenos positivos con exaltación de la "reacción de oposición", cuya versión clínica toma el aspecto negativo de disminución de la pasividad, con exageración de los reflejos locales de postura; fenómenos negativos con disminución de la reflectividad de actitud (Foix y col.), del tono de orientación (André-Thomas), del tono de expresión, hipomimia (André-Thomas) y de la motilidad automática (balanceo de los brazos al andar) y voluntaria.

La disminución de la reflectividad de actitud se expresa por una debilitación de la respuesta a la maniobra del empujón. La disminución de la motilidad automática $\mathrm{y}$ voluntaria $\mathrm{y}$ de las formas citadas de tono muscular, comporta un empobrecimiento motor por freno, por bloqueo, esto es, un empobrecimiento motor debido al entorpecimiento creado por la misma hipertonia miotática plástica. Esta es la base de la hipocinesia secundaria de los parkinsonianos. Más adelante veremos que puede existir también una hipocinesia primaria.

2) Distonias extrapiramidales - Es difícil apreciar el alcance y sentido exactos del término distonia. Frente a autores que califican así a simples aumentos paroxísticos del tono muscular, Riser y col. prefieren limitar la calificación de distonía a transtornos más complejos que comportan va- 
riaciones del tono simultáneas o sucesivas de tal o cual grupo muscuĩar, sin sistematización $y$ sin ritmo. En la acepción más adecuada de este vocablo se sitúan una serie de estados que guardan una relación de dependencia patogenética con disturbios del sistema extrapiramidal.

a) El torticolis espasmódico - A despecho del enfermo, la cabeza es conducida lentamente a la posición viciosa, no por una sola contracción. sino en dos o tres tiempos; no suele permanecer fijada, irrevocablemente desde entonces, en tal posición anómala; en un momento dado parece que la hipertonia cede bruscamente y entonces la cabeza tiende a la posición normal, pero pronto el espasmo reaparece, reiterándose así el juego de una serie de oscilaciones lentas, desiguales, en interconexión con el esfuerzo del enfermo que, conscientemente o no, se opone a esta secuencia que le atormenta. Un detalle clínico curioso es el frecuente gesto de apoyo por el cual el paciente tiende a amortiguar la distonía. Su forma más frecuente es el apoyo de la mano en la mandíbula o mejilla. Puede ser substituído a veces con igual eficacia por el mismo apoyo realizado por el observador.

No puede afirmarse el carácter extrapiramidal de todo torticolis espasmódico. Otros factores, muy principalmente psicógenos, han de ser tenidos en cuenta.

b) Los espasmos segmentarios - Trata-se de distonias localizadas a una extremidad, con las mismas características distónicas del torticolis espasmódico. Se trata, dice Riser, de un movimiento de torsión del miembro sobre su eje, de pronación y supinación forzadas, a veces alternantes. La contracción es habitualmente lenta y progresiva. Es frecuente la contracción simultánea de músculos antagonistas. Estas manifestaciones distónicas pueden asociarse a otras más bien discinéticas, a veces a sintomas piramidales, a síntomas cerebelosos, a defectos sensitivos y aún a hemianopsia. Las alteraciones del tono muscular son entonces de lo más abigarrado.

Garcin ha descrito un interesante aspecto semiológico de la mano distónica, sometiéndolo a un detallado análisis. Se trata del llamado "signo de la mano excavada o hueca", debida a una hipertonía de los músculos responsables de la flexión-aducción del primer metacarpiano. La expansión aponeurótica que el abductor corto envía al tendón del extensor largo del pulgar explica la constante extensión de la segunda falange de éste en tales corcunstancias. Según comenta Garcin, tal "mano excavada tónica" no es más que un fragmento de atetosis o el primer grado - precediendo a la pronación - del movimiento de torsión y de arrollamiento del miembro superior que se observa en buen número de afecciones de los ganglios basales. Para explorar este fenómeno, se le pide al paciente que coloque los antebrazos flexionados verticalmente, con la palma de las manos hacia delante y los dedos extendidos y separados entre si, procurando mantener el primer metacarpiano en el mismo plano que los demás.

c) La distonía de torsión - Según André-Thomas y De Ajuriaguerra, atendiendo a la expresión capital de la distonia en la musculatura del eje corporal, los fenómenos que la integran se presentan en dos formas: ei 
espasmo de torsión intermitente, que imprime momentáneamente al eje actitudes bizarras de inclinación, torsión, anteflexión, retroflexión; la distonía permanente, que aparece en cuanto se adopta la actitud bípeda o la marcha, aún la posición sentada y que desaparece en el reposo en el decúbito dorsal. Ligada a la actividad, la distonía toma un aspecto especial según el tipo de ésta, de tal modo que al pasar de la estación bípeda a la deambulación, la disbasia añade una serie de movimientos o de actitudes estereotipadas del tronco y de las extremidades, que se renuevan en cada tiempo de la marcha, de manera que la progresión y las sinergias que ella comporta producen una mutación de la distonía.

La supeditación de la distonía a la actitud bipeda en algunos casos, conocida desde las descripciones de Oppenheim, autoriza a emplear más selectivamente para ellos el término de "distonías de actitud", sobre el que Thevenard forjó su tesis. Para este autor tal distonía derivaría esencialmente de una hipotonía de los músculos del plano posterior, de los que normalmente se suspende el indivíduo durante la estación bípeda (concepción de la estación bípeda relejada como un "equilibrio en suspensión", quedando la gravedad por delante y el tono suspensor de actitud por detrás, debida a Foix y su Escuela), abandonándose a este automatismo de actitud. De entre los músculos del plano posterior serían los sacrolombares los más afectados.

La aparición de la distonía ligada a cierta actitud recuerda aquellos otros casos em que el transtorno aparece ligado también a una función determinada, cual es el caso de los "calambres profesionales", en los cuales los factores psicógenos deben ser tenidos en cuenta.

Como hemos visto anteriormente, para Denny-Brown existe una transición desde las dos posturas básicas de la atetosis, a través del espasmo móvil alternante (distonía muscular deformante), hacia las distonías fijadas según patrones característicos.

3) Posible hipotonía y transtornos abigarrados del tono muscular Una disminución del tono muscular ha sido considerada como propia de lesicnes del striatum. En la corea benigna o de Sydenham la hipotonía tiene el aspecto clínico cerebeloso, con aumento de la pasividad; la afectación del sistema cerebeloso (de los pedúnculos superiores, p. ej.), a uno u atro nivel, es perfectamente sostenible.

Ya nos hemos referido a posibles cuadros clínicos con transtornos abigarrados del tono muscular. Así ocurre en los síndromes rubro-subtalámicos, en el síndrome de la encrucijada hipotalámica en donde pueden combinarse discinesias, aumento de la pasividad, hiperextensibilidad.

C) Hipocinesia primaria - Nos hemos referido ya al efecto lentificador de la hipertonia sobre la motilidad voluntaria. Por este camino hemos visto que se explicaba gran parte de la hipocinesia de los extrapiramidales, hipocinesia que habia que considerar como secundaria. Es la hipocinesia que puede mejorar al aliviarse (p. ej. quirúrgicamente) la rigidez parkinsoniana. Pero puede haber también otro componente hipocinético que no 
mejora por este caminho (Housepian y L. Pool, Mettler y col.). Es una hipocinesia extrapiramidal a la que cabe denominar primaria, en oposición a la anterior.

D) Finalmente, queda por citar un hecho notable, la denominada cinesia paradójica (Souques, Babinski y Jarkowski y otros) de algunos pacientes extrapiramidales, consistente en la facilidad y desahogo con que el sujeto realiza ciertos actos motores, complejos o dificiles (bailar, p. ej.), mientras que, por el contrario, su motilidad habitual está profundamente alterada, sea por transtornos de tipo parkinsoniano, sea por disturbios extrapiramidales de otra serie.

\section{LOS MECANISMOS FISIOPATOLOGICOS}

A la nitidez, no exenta de complejidad, de los hechos clínicos, se opone, en el campo de la patologia extrapiramidal, la inseguridad de las hipótesis fisiopatológicas.

Como ha comentado Walshe, la mayoría de tratadistas se precipitan en querer interpretar el sindrome observado en función de la topografía de las lesiones en juego, antes de enjuiciar la fenomenología clínica de aquél como expresión de un disturbio en el desempeño de unas determinadas funciones.

Walshe discrimina tres pasos sucesivos que deberían recorrerse para construir un razonamiento cabal de los sintomas esenciales del síndrome parkinsoniano, actitud que puede generalizarse para la totalidad de fenómenos clínicos extrapiramidales: $a$ ) observación de las características de estos síntomas y precisión de sus relaciones con el tono y los movimientos del estudo normal, es decir, su análisis funcional; b) correlación de estos trastornos con las lesiones nerviosas que condicionan su aparición, procurando alcanzar así una localización lesional más o menos perfecta; c) tentativa de interpretar el trastorno funcional y la localización lesional, con la intención de procurar aclarar el papel que ejercen en el control del tono $\mathrm{y}$ del movimiento las estructuras dañadas $\mathrm{y}$ las que permanecen intactas. "Por ser este tercer paso, la denominada localización de la función, un proceso de inferencia lógica - comenta Walshe — es por lo que es tan difícil, y su dificultad se ve aumentada por las imperfecciones de nuestro conocimiento de la anatomía normal y patológica y de nuestro conocimiento fisiológico".

De acuerdo con esta posición de Walshe, lo primero que hay que hacer ante un fenómeno clínico extrapiramidal, no es intentar situarlo dentro del complejo diagrama de unas conexiones anatómicas, como simple consecuencia de la interferencia lesional con una supuesta acción estimuladora o inhibidora de uma estructura sobre otra, sino plantearse sus relaciones con los fenómenos que informan la tonicidad y la motórica en estado normal. Si, además, puede irse aclarando de algún modo la relación entre las bases funcionales de lo que es normal y el disturbio fisiopatológico que preside el fenómeno patológico, la visión propuesta gañará en penetración y finura. 
Una actitud que sigue hasta cierto punto los postulados señalados por Walshe, es la que ha ido estructurando Denny-Brown en una serie de trabajos sobre fisiopatologia extrapiramidal y otros trastornos. De acuerdo con Denny-Brown hay que ver en la hipercinesia, en la discinesia y en la distonia extrapiramidales la consecuencia de la rotura de alguna forma de equilibrio que normalmente interviene en la modulación del tono, de la postura y del movimiento. El sintoma expresa el sentido de esta rotura, su intimidad fisiopatológica, y puede ayudar a penetrar la organización funcional normal tanto más si es conocida la distribución de las lesiones en juego.

Para seguir la concepción de Denny-Brown hay que empezar por recordar que numerosos campos corticales poseen proyecciones extrapiramidales, incluso posiblemente las cuatro áreas que, según Woolsey, dán origen al sistema piramidal.

Según Denny-Brown, en estado normal existiria un equilibrio balanceado entre modulaciones corticales de la actividad motora de tipo positivo (exploratorio) y negativo (evitativo), tanto en la esfera de las aferencias táctiles como en la de las visuales. En la esfera táctil, ligeras reacciones de evitamiento coexistirian y alternarian con reacciones de exploración, tales como la reacción de posición. Este equilibrio se basaría en el interjuego establecido entre las regulaciones regidas por las áreas de función positiva y aquellas otras dependientes de las de función negativa.

Denny-Brown, por lo demás, tiene el mérito de subrayar el nexo que aúna las actividades sensitivas con las motoras del sistema nervioso, esto es, las aferencias con las eferencias. Ambas aparecian excesivamente distantes entre si en los esquemas expositivos clásicos, cuando la realidad es muy otra, según puede mostrarnos la simple observación sumada a la meditación más somera. Aferencias y eferencias trabajan para un conjunto que vive actuando en relación con el medio ambiente, estableciendo con el una relación transaccional que von Weiszaecker anticipó en el concepto de "círculo figural". En realidad, como ha insistido justamente P. Lain Entralgo, el desplazamiento en la dimensión temporal de este sistema, hace más apropiada la calificación de "espiral" que la de "círculo" y, como el mismo autor subraya, en el caso particular del hombre, el ejercicio de su libre voluntad rompe una y otra vez dicho sistema.

Aplicando sus concepciones sobre la desintegración de las actividades motoras por rotura de los equilibrios aferentes-eferentes a las disfunciones extrapiramidales, Denny-Brown mantiene las siguientes hipótesis: 1) en la corea habría un desequilibrio en los automatismos corticales; 2) en la atetosis y en la distonía habría una liberación inestable y alternante de elementos subcorticales; 3 ) el temblor representaría un desequilibrio más violento entre extremos positivos y negativos.

Para precisar estas hipótesis sobre la naturaleza de los disturbios extrapiramidales, Denny-Brown elabora sus concepciones acerca de los níveles cortical y subcortical de integración de las respuestas en la siguiente forma: el nivel cortical sería el más alto y fino y se estructuraría de acuerdo con 
la exposición antes resumida; la organización subcortical se disponía según tres niveles sucesivos. En el striatum, principalmente en el putamen, se establecería una elaboración de las respuestas integradas a nivel inferior en relación con las reacciones contactuales y visuales, merced a las conexiones córticostriadas. Así pues, el striatum prolongaría las modulaciones de origen táctil y visual en las que intervendría decisivamente la corteza cerebral. Recordemos, por lo demás, la acción de signo frenador o inhibidor que, en general, suele otorgarse a este nivel extrapiramidal. El nivel tálamopalidal favorecería las respuestas de enderezamiento de origen laberíntico, tendiendo a provocar una extensión, al menos de las extremidades inferiores, permitiendo su conflicto distónico con las reacciones contactuales originadas en el cuerpo, explicando así las modificaciones de la postura que experimentan algunos pacientes al variar de decúbito. A nivel subtalámico tendria lugar una combinación de efectos cerebelosos (vía del pedúnculo cerebeloso super:or) y propioceptivos, produciéndose respuestas reflejas de enderezamiento del cuerpo sobre el cuerpo.

Los procesos morbosos, al producir la lesión electiva o preferente de los varios niveles cuya función respectiva quedaría asi esquematizada, determinarian una desintegración de las funciones motoras por diferentes modos de rotura de los mecanismos de integración, liberándose varios tipos de respuestas que, además, podrían entrar en conflicto entre sí. Veamos como ocurriría esto, siempre según Denny-Brown, en las lesiones del striatum, del pallidum y de la substancia reticulada subtalámica.

A. Lesiones del striatum - La lesión del núcleo caudado liberaría una profusión de automatismos corticales, dando lugar a un cuadro coreico. Orientando hacia sus concepciones la idea general de una función inhibidora de esta estructura, Denny-Brown sugiere que el núcleo caudado actuaria normalmente como supresor de un grupo de respuestas corticales mientras otro grupo está en actividad. La pérdida de esta supresión fisiológica comportaría el conflicto hipercinético descrito como corea.

La lesión progresiva del striatum o solamente del putamen se manifestaría en su primera fase por la atetosis. Esta hipercinesia derivaria de la liberación simultánea de respuestas tónicas de prensión y de evitamiento, frecuentemente en conflicto con las respuestas visuales correspondientes, Podría, pues, decirse que la atetosis no tendría un contenido positivo, que no seria nada en sí mismo, sino que representaría solamente el conflicto constante entre respuestas tónicas de diferentes tipos. Al aumentar las lesiones, se incrementaría la liberación de las respuestas integradas a nivel inferior, esto es, de respuestas laberinticas y de respuestas de enderezamiento por contacto. La atetosis tendería asi a evolucionar hacia la distonia fijada. La distonía muscular deformante representaría una situación intermedia en esta evolución. El estado final distónico comportaria primeramente una actitud en flexión de las extremidades superiores y en extensión de las inferiores (d.stonia hemip.éjica de Denny-Brown) que podría ser sub tituída ulteriormente por una flexión de todas las extremidades. Sin embargo, débido a la liberación de las respuestas laberínticas, al sus- 
pender el paciente, las extremidades inferiores se extenderían. La lesión brusca del striatum (infarto primario del putamen y del núcleo caudado) determinaría una "distonía hemipléjica" inmediata.

B. Lesiones del pallidum - En los casos de evolución progresiva apareceria un temblor que podria evolucionar hacia una distonía con flexión generalizada, que no se modifica al suspender el paciente. Este detalle se explicaría porque la lesión palidal interferiría con los circuitos tálamopalidales en relación con respuestas de enderezamiento de origen laberíntico. En las lesiones bruscas (por ejemplo, en la necrosis palidal bilateral de la intoxicación por monóxido de carbono) se instauraría inmediatamente la distonía en flexión generalizada, acompañada de 'mutismo aquinético.

C. Lesiones de la substancia reticulada subtalámica - En las lesiones parciales se produciria un temblor proximal rotatorio. Denny-Brown subraya también el componente rotatorio, del hemibalismo. En lesiones más extensas apareceria una actitud de hemiplejía espástica con ratación interna de los miembros. Esta actitud representaria el tipo clínico de rigidez por descerebración, cuya forma experimental (subrúbrica, intercollicular) fué descrita por Sherrington, consistiendo ésta en una actitud generalizada de hipertonia antigravitatoria de predominio proximal y sin rotación interna. Añadamos que la lesión del núcleo subtalámico de Luys se considera como la responsable de la aparición del hemibalismo, si bien Dierssen y Gioino, revisando detalladamente la literatura, han puesto de manifiesto el carácter inconstante y relativo de esta correlación.

La argumentación de Denny-Brown tiene el gran mérito de haber resaltado el nexo que aúna $y$, hasta cierto punto, unifica las diversas hipercinesias y distonías extrapiramidales, así como la de haber contribuído a situar estos trastornos del movimiento, de la postura y del tono en el seno del "espiral figural" por el que discurren las funciones motoras. Además, sus descripciones y muchas de sus hipótesis ayudan indirectamente a hacer más visible la complejidad de la motilidad normal, tejida sobre una sutil y finísima trama de adaptaciones moduladas por las estimulaciones sensitivas y sensoriales, sobre las cuales el imperio de los movimientos voluntarios viene a ser más una "apertura" que una "rotura", si resulta afortunada esta manera de expresarse. Queremos decir con ello que la motilidad voluntaria no solamente requiere el apoyo firme que le presta una "motilidad basal" (en cierto sentido, la motilidad ereismática de Hess), sino que en su ejecución no dejan de intervenir las regulaciones que, derivadas de multitud de aferencias, dán lugar a finos ajustamientos involuntarios imprescindibles. De estos hechos la Neurología clásica conocía bien la influencia de la sensibilidad profunda de cuya pérdida resulta ataxia. Pero este es sólo un ejemplo de una serie mucho más amplia. La patología extrapiramidal, al quebrar la rotundidad $y$ tersura de las adaptaciones normales, condiciona la "parasitación" de la motilidad por una serie abigarradisima de hipercinesias, de discinesias, de distonias, resultantes de complicados procesos de liberación competitiva que expresan la indole de la desorganización existente. Es la reducción a la fisiologia de estos razonamien- 
tos fisiopatológicos sobre una semiología tán detonante, la que nos permite columbrar la suprema complejidad y sutileza que debe poseer la organización del desempeño motor en condiciones de normalidad.

Finalizaremos nuestro estudio de la fisiopatología de los trastornos motores extrapiramidales, completando esta exposición de las hipótesis de Denny-Brown con una visión sumaria de lo que parece ser el mecanismo de la rig:dez parkinsoniana y con un planteamiento de los factores que posiblemente intervienen en la génesis del temblor de reposo y de las cinesias paradójicas.

La rigidez parkinsoniana traduce una exaltación plástica de la "reacción de oposición", del antagonismo miotático. En el origen de esta exaltación plástica juega probablemente un papel decisivo (Matthews y Rushworth, Barraquer-Bordas, Rushworth) cierta forma de hiperactividad del sistema gamma de inervación intrafusal. El aumento de los reflejos locales de postura es una manifestación más de la rigidez. El fenómeno de la rueda dentada expresaría la sucesión de una serie de reacciones de acortamiento (exageradas por la hiperactividad gamma) y de alargamiento. La disminución de la respuesta re-equilibratoria de actitud en la maniobra del empujón, así como del tono de orientación y de expresión y de la motilidad automática y voluntaria, serían consecuencia indirecta de la rigidez. "Todos estos procesos tónicos y cinéticos resultarían frenados poco a poco en la rigidez parkinsoniana por la cortina tendida por la liberación y exaltación plática del antagonismo miotático, cortina que vendría a interferir el curso de toda esta serie de fenómenos motores, "impermeabilizando" la vía terminal común, que iría perdiendo así progresivamente sus cualidades funcionales de "prontitud de paso" caracteristicas del estado normal" (Barraquer-Bordas). Esta impermeabilización por saturación consistiría, neurofisiológicamente, en un proceso de oclusión que bloquearía progresivamente la vía terminal común o, para ser más exactos, el circuito motoneurona gamma-bucle periférico-motoneurona alfa.

Cómo abordar el problema de la fisiopatołogía del temblor parkinsoniano? Quizás puntualizando como cuestión previa el que tal temblor en reposo prejuzga tres requerimientos: la afectación de estructuras extrapiramidales; la disfunción de sistemas descendentes que actúan sobre los níveles segmentarios; una actividad rítmica anormal de tales níveles (sea ésta o no una pura expresión de los impulsos anormales que aquí llegan).

Para Jung y para Das Gupta, el mecanismo esencial del temblor estriba en una liberación de movimientos rítmicos antagonistas estructurados en los niveles inferiores, espinales y bulbares. Concretamente, para Das Gupta existe en el Parkinson una actividad sostenida de las interneuronas espinales que mantiene un estado excitador. La opinión habitual hace depender esta situación de una estimulación retículo-espinal tónica permanente. Pero, además de esta influencia facilitadora, existiría, según Das Gupta, una acción igualmente suprasegmentaria inhibidora y también influencias inhibidoras acrescentadas de origen periférico, procedentes de los órganos 
tendinosos de Golgi (Hufchmidt). El temblor rítmico parkinsoniano depen. dería entonces del interjuego establecido a nivel de las neuronas intercalares espinales entre actividades tónicas antagonistas, comportando una oscilación del servo-sistema médulo-periférico. Según ello, el temblor parkinsoniano no requeriría la presencia de una ritmicidad análoga por parte de los influjos descendentes supraespinales.

Para Jenkner y Ward Jr., las lesiones que determinan temblor en reposo interrumpen vias que van hacia la formación reticulada, con lo que las neuronas de esta formación, asi desaferentizadas, se tornan (según una aplicación de la conocida ley de Cannon), hipersensibles a la acetilcolina producida durante la actividad normal de las neuronas vecinas. Esta hiperactividad se proyectaria por vías retículoespinales y sería la base esencial del temblor. Para Schwab, el papel de los impulsos piramidales ritmados, de fondo, normales, sobre el desajuste condicionado por la lesión extrapiramidal, podría ser decisivo.

Según Gybels, en la génesis del temblor podrían tener también un papel decisivo impulsos derivados de la actividad piramidal, pero que serían ya impulsos anormales debidos a un desajuste funcional de neuronas corticales condicionado por las lesiones de las estructuras grises basales. Según Spiegel y col., podría producirse experimentalmente temblor por estimulación de la substancia reticulada mesencefálica y pontina (lo que apoyaría la tesis de Jenkner y Ward Jr.) y este efecto se facilitaria lesionando la substancia nigra. Lesiones nígricas o yuxtanigricas pueden incluso llegar a producir temblor por sí mismas, queremos decir sin el concurso de estimulaciones (Schreiner y col.). Recordemos que para Denny-Brown el temblor es la expresión de la alternancia rítmica de un conflicto semejante al que se manifiesta en la atetosis (basado en aferencias propioceptivas), aunque más violento.

Es evidente que las hipótesis que acabamos de resumir, examinadas con cierto temple de serenidad propicio a una visión amplia y sintética, no se oponen totalmente entre sí. Lo que ocurre es que las bases lesionales de la situación son de sistematización muy difícil y que los mecanismos físiopatológicos en juego son harto sutiles y complejos. Un comentario de J. French se sitúa en esta línea. "Es perfectamente presumible - dice este autor - que influencias remotas procedentes de los ganglios basales y de estructuras relacionadas con ellos, actúan sobre la función reticular y contribuyen de modo importante a la génesis del temblor, como sugiere Ward. Casi con certeza, su energía trastormada se proyecta a la vez en sentido cefálico y en sentido caudal, para ser transmitida a las motoneuronas alfa y gamma tanto por rutas "piramidales" como "extrapiramidales". Estas influencias corticales y subcorticales sobre la función motora están altamente sufisticadas y su distorsión por la enfermedad es infinitamente complicada".

Ahora es el momento de referirse a las experiencias recientes de Hofmann que pertocan a la vez a las posibles bases de la rigidez y del temblor parkinsonianos. Tales investigaciones parec€n indicar que la situación fun- 
cional del sistema gamma está singularmente alterada en el Parkinson, si bien esta alteración no podría reducirse a un esquema cuantitativo de hipoactividad o hiperactividad. Para este autor, la disfunción de la inervación gamma intrafusal $\mathrm{y}$, por lo tanto, de los receptores neuromusculares, en los parkinsonianos es sumamente compleja. En primer lugar, la índole de esta disfunción varía según se la considere en cuanto al síntoma rigidez o en cuanto al sintoma temblor. En la rigidez parkinsoniana se habría perdido cierta fracción de la activación supraespinal del sistema gamma, el cual perdería entonces gran parte de su capacidad especifica tocante a la posibilidad de diferenciación entre la longitud de los elementos musculares extrafusales e intrafusales. Este defecto sería singularmente notorio para el ca o de los impulsos lentos, tónicos, de finalidad postural, mientras que pasaría desapercibido en lo tocante a la activación fásica, rápida, del propio sistema gamma. Tal situación afectaría considerablemente el control de gran número de influencias, tanto centrales como periféricas, destinadas a recaer sobre el sistema alfa, lo que determinaria, fuese cual fuese la longitud del músculo, un bombardeo simultáneo de las unidades motoras de agonistas $y$ antagonistas, mantenido constantemente a un nivel casi invariable, debido al fallo de actividad de inhibición reciproca propia del aparato reflejo del huso muscular cuando trabaja en condiciones normales. Las motoneuronas alfa dejarían así de ser "informadas" del momento en que las fibras musculares inervadas por ellas han alcanzado la long-tud adecuada. No se trataría, pues, según estas hipótesis de Hofmann, de una simple desaferentización de los husos, como postulaba Hassler, sino de un trastorno más complejo, de un "disturbio tan solo relativo". Los efectos de este disturbio aparentarian corresponder, como el mismo Hofmann sugiere en su examen critico del problema, a una hiperactividad gamma, aunque realmente no sería así.

Por otra parte, según este mismo investigador, al temblor parkinsoniano guardaría también cierta relación con el disturbio de la función de "servomecanismo" del rizo periférico en esta situación morbosa. La desaferentización aumenta la amplitud del temblor, la cual estaría ligada a esta situación anormal, de signo fluctuante, además de dicho "servomecanismo". Asi pues, la amplitud del temblor parkinsoniano estaría sometida a un cierto control periférico, mientras que su frecuencia vendría determinada por un mecanismo central. La sensibilidad del temblor a modificaciones posturales de efectos propioceptivos y su inhibición por la motilidad voluntaria subrayan - afirma Hofmann — de acuerdo con la opinión clínica tradicional, la probable independencia mutua de los mecanismos responsables de la rigidez y del temblor en el Parkinson.

El aumento del temblor obtenido por desaferentización que menciona Hoimann se correlaciona con el efecto contrario logrado por Subcynski, Matsumoto, Lin y Cooper mediante la maniobra opuesta. En efecto, según estos autores, la estimulación propioceptiva tiene una influencia inhibidora sobre el temblor.

Cooper y col. subrayan que el temblor y la rigidez se comportan de modo diferente ante diversos estímulos. La tensión mental produce un 
aumento casi aislado del temblor. La alteración de la información propioceptiva parece ser más importante en el temblor que en la rigidez. Los movimientos contralaterales activos exageran la rigidez, lo cual nos recuerda las sincinesias de imitación y el "signo de la muñeca fijada" de Froment y Garcin.

Nos hemos referido antes, muy de paso, a una posible hipocinesia primaria en el Parkinson, no dependiente de la rigidez. La realidad de esta hipocinesia viene avalada por la experimentación (Escuela de Mettler)y por el escrutinio de los resultados operatorios (Housepian y L. Pool). Según Denny-Brown tal hipocinesia podría explicarse por una interferencia con mecanismos facilitadores de circuito tálamopalidal. Este autor concede un gran valor a las integraciones regidas por este circuito.

Un capítulo singularísimo y muy peculiar de la semiología extrapiramidal está constituído por los fenómenos que se agrupan bajo la etiqueta de cinesia paradójica. Se trata, en realidad, de hechos desiguales entre sí. Unas veces son movimientos sorprendentes pero en sí mismo anormales (la arrojada carrera de un parkinsoniano habitualmente casi "estatuario"), mientras que en otras ocasiones llega a ser la aparición inesperada de un comportamiento expresivo motor normal, favorablemente influido, al menos en algunas ocasiones, por una situación emocional concreta. Aunque habitualmente los componentes más automáticos de la motórica son los más afectados, los más entorpecidos, no siempre es asi, como puede observarse en los bloqueos de los movimientos de la mirada (Jarkowski, Subirana).

Denny-Brown ha comentado el papel opuesto que juegan las regulaciones visuales en diferentes tipos de cinesia paradójica. Así, por un lado, en algunas distonías moderadas y en ciertos casos de Parkinson, el estímulo visual ayuda a romper el bloqueo del movimiento. En cambio, en el torticolis espasmódico existiría una liberación de las respuestas optocinéticas mantenidas por un mecanismo laberíntico-hiperactivo, debido al fallo de las reacciones de enderezamiento óptico y de fijación del cuerpo. En estas condiciones las reacciones corporales de origen táctil pueden contrarrestar el desequilibrio existente. En tales casos un ligero contacto sobre el cuello o la cabeza, del lado hacia el cual el sujeto gira la cabeza, modifica favorablemente la distonía hasta un grado aún sorprendente. Esta maniobra es aprendida por el paciente que la realiza por sí mismo. Asimismo el descanso sobre el lecho o el apoyo en estación bipeda contra una pared (DennyBrown), así como la presión ejercida sobre el occipucio por nuestras manos (Barraquer-Bordas), contrarrestan la distonía. También hemos visto mejorar ésta al tiempo que el paciente abre la boca.

Es muy notable que tanto el sueño, situación en la que los sistemas reticulares entran en reposo, como situaciones caracterizadas por una estimulación supranormal (Jarkowski), en las que es de prever una hiperactividad reticular, comportan una disminución de la sintomatología parkinsoniana o, más concretamente, de la hipocinesia. Esta aparente contradicción debe explicarse verosímilmente a expensas de las modificaciones funcionales 
de orden diferente y aún opuesto, regidas por los distintos componentes de los sistemas reticulares y por sus conexiones aferentes y eferentes (Jouvet, Batini y col., Cordeau y Mancia, Bloch y Bonvallet), así como por la intervención en la descarga motora final sea de las motoneuronas alfa fásicas, sea de las tónicas. Teniendo en cuenta que los trabajos de Granit han puesto de manifiesto que los impulsos puestos en marcha por el sistema gamma recaem, al menos preferentemente, a través del bucle periférico, sobre las motoneuronas alfa tónicas, y considerando la existencia en el Parkinson de una disfunción verosímil del sistema gamma, puede sugerirse (Barraquer-Bordas) que, en algunas formas de cinesia paradójica, los movimientos se ejecutarian principalmente a través de las motoneuronas alfa fásicas, escasamente interferidas en su función.

Lauría 102 - Barcelona - Espanha. 